International Journal of Pure and Applied Mathematics

Volume 98 No. 2 2015, 275-289

ISSN: 1311-8080 (printed version); ISSN: 1314-3395 (on-line version)

url: http://www.ijpam.eu

doi: http://dx.doi.org/10.12732/ijpam.v98i2.9

ijpam.eu

\title{
ANALYSIS OF BISTABLE AND CHAOTIC PIEZOELECTRIC ENERGY HARVESTING DEVICE COUPLED WITH DIODE BRIDGE RECTIFIER
}

\author{
C.H.C.C. Basquerotto ${ }^{1}$, F.R. Chavarette ${ }^{2}$, S. da Silva ${ }^{3}$ \\ ${ }^{1,3}$ Departamento de Engenharia Mecânica \\ Faculdade de Engenharia de Ilha Solteira \\ UNESP - Univ. Estadual Paulista \\ ${ }^{2}$ Departamento de Matemática \\ Faculdade de Engenharia de Ilha Solteira \\ UNESP - Univ. Estadual Paulista \\ Campus de Rio Claro, BRASIL
}

\begin{abstract}
Vibration energy harvesting has greatly expanded over the last few years. While linear vibration-based energy harvesting has received considerable attention in the literature, some current research is focused on the concept of purposeful inclusion of nonlinearities for broadband transduction. The energy harvesting process is performed in two steps: energy extraction and using this energy to feed electronic devices. Thus, this work discusses the use of an energy extraction device that is a chaotic non-linear mechanical device, coupled to a half-wave rectifier circuit to transform the alternating voltage generated by the piezoelectric ceramics into continuous voltage to power electronic devices. An analysis of the dynamic interaction between the two devices is done and it can be concluded that it is possible to use a mechanical device that operates in chaos coupled to a rectification circuit.
\end{abstract}

AMS Subject Classification: 74Fxx, 74Mxx, 37Mxx

Key Words: energy harvesting, piezoelectric materials, nonlinear system, chaos, half-wave diode bridge

Received: October 23, 2014

(C) 2015 Academic Publications, Ltd.

${ }^{\S}$ Correspondence author url: www.acadpubl.eu 


\section{Introduction}

The conversion of ambient vibrations into electricity with piezoelectric materials is one of the most useful solutions to convert mechanical energy in electrical energy for various wireless electronic applications [1,2]. Among these harvesting devices, the use of nonlinear devices has been widely studied to extract energy from wideband non-harmonic vibration sources $[3,4,5,6,7]$. In this sense, the use of the structure investigated by Moon [17], composed by a nonlinear device that can present chaotic behavior, is one of the most used for energy harvesting purposes in recent years using only an electric resistance to dissipate the energy generated by the piezoelectric materials $[6,7,8,9,10,18,19]$.

However, the harvesting energy from vibration sources using piezoelectric materials produces an alternate voltage. To use this energy to feed electronic devices requires converting it into direct voltage [16]. Thus, the use of rectifier circuits are required $[11,13]$, but until now, few studies have shown the dynamic interaction of a nonlinear energy harvesting device operating in chaos coupled with a rectifier circuit, mainly a basic half-wave diode bridge $[14,15,16]$.

Naik et al. [14] proposed a wideband energy harvesting using a combination of an optimized synchronous electric charge extraction circuit and a bi-stable harvester. The results showed that with these two nonlinear devices, the harvester device operates more efficiently, hence more output power can be obtained in a broad frequency range. Chen et al. and Liu et al. [21, 22] presented a novel energy harvesting solution: the optimized synchronous electric charge extraction (OSECE) with a bistable buckled-spring-mass (BSM) that can be chaotic for certain frequency values and that can lead to high efficiency energy harvesting. These nonlinear techniques enable the piezoelectric harvester to work more efficiently, hence providing more output power at a broadened frequency range [21]. Clementino et al. [25] presented their work in the dynamic interaction of a linear mechanical device coupled to a rectifier circuit.

Most studies in this area do not show the interaction between a mechanical device coupled with a rectifier circuit. So, the main goal of this work is to analyze the dynamical behavior of a chaotic bi-stable harvesting device combined with a half-wave diode bridge. Numerical tests were performed and the results presents the dynamic interaction between these two devices showing that the mechanical device continues to operate in chaos when coupled to a rectifier circuit and that it is possible to rectify the chaotic voltage. This paper is organized in five sections as follow. Firstly, a brief review of the classical dynamic of a bistable chaotic energy harvesting device used for energy harvesting. Next, the half-wave diode bridge rectifier equations are illustrated. Finally, the numeri- 
cal results of the interactions between the nonlinear harvester and the rectifier circuit and the concluding remarks are presented.

\section{Nonlinear Energy Harvesting Device Coupled with Rectifier Circuit}

The nonlinear device is an aluminum cantilevered beam $145 \mathrm{~mm}$ length $25 \mathrm{~mm}$ width and $0.26 \mathrm{~mm}$ thickness with two magnets located symmetrically near the free end, as seen in Figure 1a. The device is subjected to harmonic base excitation given by Acoswt. A lumped mass of 14 grams is attached to the free end of the ferromagnetic beam tip to improve the dynamic flexibility and to allow the motion caused by the magnets. Therefore, depending on the magnet spacing, the ferromagnetic beam may have five (with three stable), three (with two stable) or one (stable) equilibrium positions [7, 8, 16, 17].

The equations that describe the device for the condition with three equilibrium positions for the fundamental vibration mode are $[8,9,10]$ :

$$
\begin{gathered}
\ddot{x}+\frac{c}{m} \dot{x}-\frac{k_{l}}{m} x+\frac{k_{n l}}{m} x^{3}-\frac{q}{m} v_{p}=A \cos \omega t \\
c_{p} v_{p}+\theta \dot{x}=-i_{p}
\end{gathered}
$$

where $m$ is the equivalent mass of the beam, $x$ is the displacement of the beam in the transverse direction, $c$ is the damping, $k_{l}$ is the equivalent linear stiffness of the beam calculated by the finite element method (FEM), the term $k_{n l}$ provides a nonlinear restoring force at large $x$ caused by the spacing of the magnets $[8,9,10,17,18,19]$. The signal of the parameters $k_{l}$ and $k_{n l}$ define the type of spring. For $k_{l}<0$ and $k_{n l}>0$ is defined as the potential energy of the system with two points of minimum and maximum point [23], $v_{p}$ is the piezoelectric voltage, $A$ is the excitation force amplitude due to base acceleration, $\omega$ is the excitation frequency and $i_{p}$ is the electric current in the piezoelectric that is crossing the rectifier circuit. The electromechanical coupling coefficient is given by:

$$
\theta=-e w_{p}
$$

where $e$ is the dielectric permittivity and $w_{p}$ is the width of the PZT [16]. The piezoelectric capacitance is given by:

$$
c_{p}=2 \cdot \frac{w_{p} l_{p} \epsilon^{S}}{t_{p}}
$$




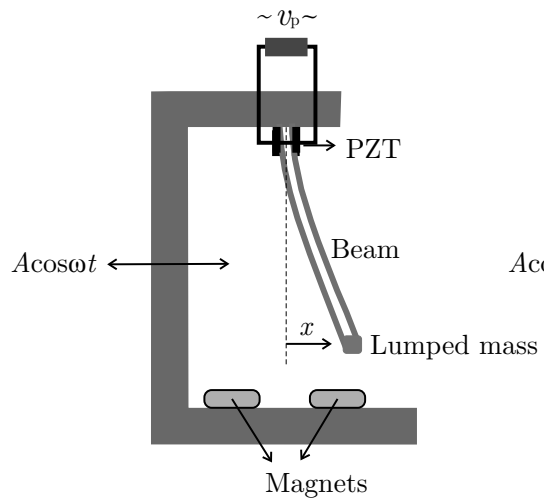

a) Nonlinear energy harvesting device.

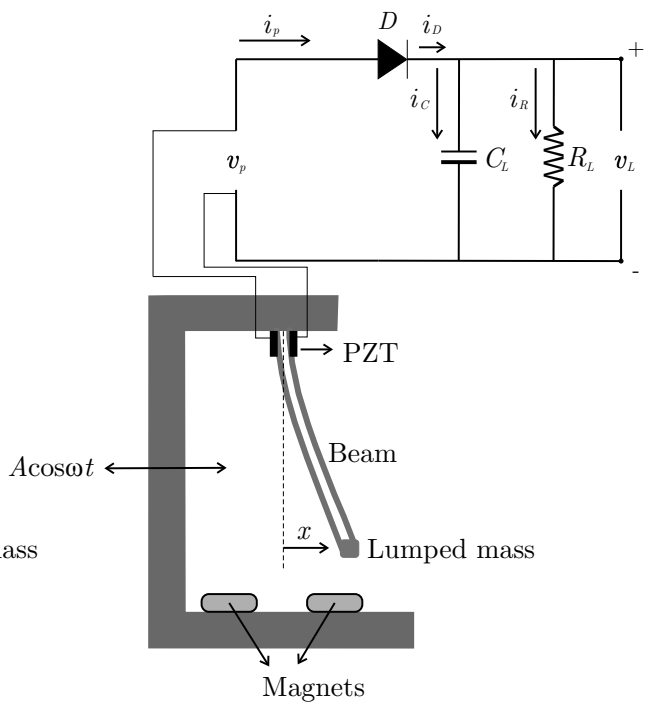

b) Nonlinear energy harvesting device coupled with the rectifier circuit.

Figure 1: Nonlinear energy harvesting device.

where $l_{p}$ is the length of the PZT, $\epsilon^{S}$ is the matrix dielectric constants for constant mechanical deformation and $t_{p}$ is the thickness of the PZT [16].

Figure $1 \mathrm{~b}$ shows a circuit that rectifies the PZT alternate voltage through a half-wave diode bridge with a smoothing capacitor filter $C_{L}$ and resistive load $R_{L}$. The voltage $v_{p}$ and current $i_{p}$ are the voltage and current of the piezoelectric device, respectively.

According to [12], systems are coupled assuming a condition of continuity between current and voltage of both:

$$
\begin{aligned}
v_{\text {circuit }} & =v_{P Z T} \\
i_{\text {circuit }} & =i_{P Z T}
\end{aligned}
$$

The current through the capacitor $\left(C_{L}\right)$ is called $i_{C}$ and $i_{R}$ and the current through the resistive load $R_{L}$. Therefore, by Kirchhoff's laws:

$$
i_{p}=i_{C}+i_{R}=0
$$

In the rectification cycle, when the capacitor is discharging, there is no current passing through the diode $\left|v_{p}\right|<v_{D}+v_{L}$. Where $v_{D}$ is the voltage drop across the diode and $v_{L}$ the resistive load voltage. The current of piezoelectric 
layers is defined by:

$$
i_{p}=C_{L} \dot{v_{L}}+\frac{v_{L}}{R_{L}}=0
$$

in the positive half cycle $\left|v_{p}\right|>v_{D}+v_{L}$, the current of piezoelectric layers is defined by:

$$
\begin{gathered}
i_{p}=i_{C}+i_{R}=C_{L} \dot{v_{L}}+\frac{v_{L}}{R_{L}} \\
i_{p}=\frac{v_{p}-v_{D}-v_{L}}{R_{D}}
\end{gathered}
$$

where $R_{D}$ is the internal resistance of the diode.

\section{Numerical Tests of the Mechanical Device}

This section presents the numerical results used to illustrate the proposal of this work. The variable step Runge-Kutta method was used for the resolution of ordinary differential equations. The results are divided in two parts: first, tests were done with only the mechanical device coupled to an electrical resistance. In the second one, the rectifier circuit was coupled with the mechanical device.

\subsection{Mechanical Device Coupled to an Electrical Resistance}

Table 1 shows the values used in the parameters of the nonlinear energy harvesting, which are the same properties of the nonlinear device studied in [18, $19,9,10]$.

After solving the equations (1) and (2) the following tests were performed: the generated voltage analyzer to two different initial conditions and the behavior of the device was verified. In order to provide these tests, the amplitude and frequency excitation were chosen as $0.5 \mathrm{~g}$ and $8 \mathrm{~Hz}$, respectively, based on $[18,19,9,10]$. Figure 2a shows the high voltage produced by the PZT and Figure $2 \mathrm{~b}$ shows the phase space of displacement versus velocity.

If the excitation amplitude is increased the system fails to present a chaotic behavior and displays a periodic regime as seen in Figure 3 (this can be proven by calculating the Lyapunov exponents), beyond the level of energy produced is less with an amplitude three times higher $(A=1.5 \mathrm{~g})$.

Figure $4 \mathrm{a}$ shows that the system is chaotic because there are infinite points in plot $x$ versus $\dot{x}$. On another hand, Figure $4 \mathrm{~b}$, for conditions $A=1.5 \mathrm{~g}$ and $\omega=50.27 \mathrm{rad} / \mathrm{s}$, the system is regular because there is only one point.

Figure 5 shows the Lyapunov exponents computed by the Wolf method from time series [24]. It is clearly observed that the device operates in chaos. When 
Table 1: Coefficients in constitutive relations.

\begin{tabular}{cccc}
\hline Constitutive relation & Nomenclature & Value & Unit \\
\hline Equivalent mass & $m$ & 0.0155 & $\mathrm{~kg}$ \\
Mass of the beam & $m_{b}$ & 0.0078 & $\mathrm{~kg}$ \\
Mass of PZT & $m_{p}$ & 0.0019 & $\mathrm{~kg}$ \\
Damping & $c$ & 0.0041 & $\mathrm{Ns} / \mathrm{m}$ \\
Linear stiffness & $k_{l}$ & 2.72 & $\mathrm{~N} / \mathrm{m}$ \\
Nonlinear stiffness & $k_{n} l$ & 3.0 & $\mathrm{~N} / \mathrm{m}^{3}$ \\
Piezoelectric coupling & $\theta$ & 0.38 & $\mathrm{~N} / \mathrm{V}$ \\
Piezoelectric capacitance & $c_{p}$ & $1.25 \times 10^{-11}$ & $\mathrm{~F}$ \\
Resistance of the load & $R_{L}$ & 1000 & $\Omega$ \\
Frequency & $\omega$ & 50.27 & $\mathrm{rad} / \mathrm{s}$ \\
\hline
\end{tabular}

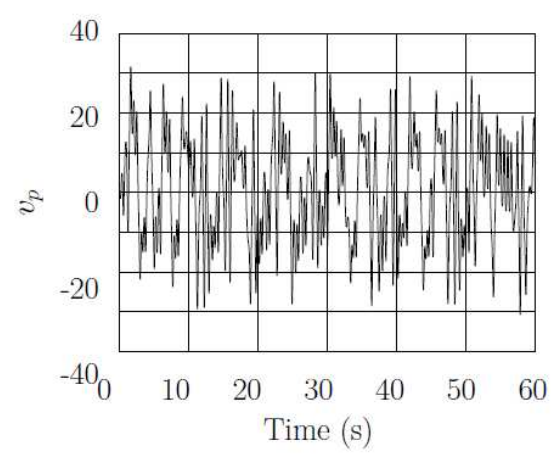

(a) Time voltage in PZT.

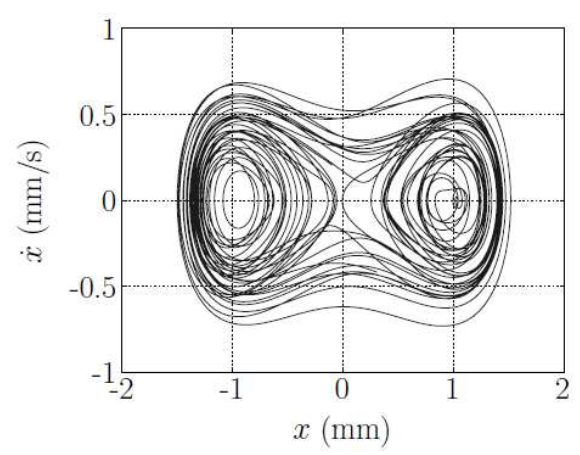

(b) Displacementxvelocity.

Figure 2: Theoretical analyses with $A=0.5 \mathrm{~g}$ and $\omega=50.27 \mathrm{rad} / \mathrm{s}$.

the system has as amplitude of excitation between $0.3 \mathrm{~g}$ and $1 \mathrm{~g}$ the device is chaotic. For $A=1.5 \mathrm{~g}$ the device is not chaotic.

\subsection{Mechanical Device Coupled to the Rectifier Circuit}

The rectifier is coupled to the mechanical device and Table 2 shows the parameters used in the rectification circuit based on [?]. When the rectifier circuit is coupled to the mechanical device, are solved the equations (1) and (2) with the 


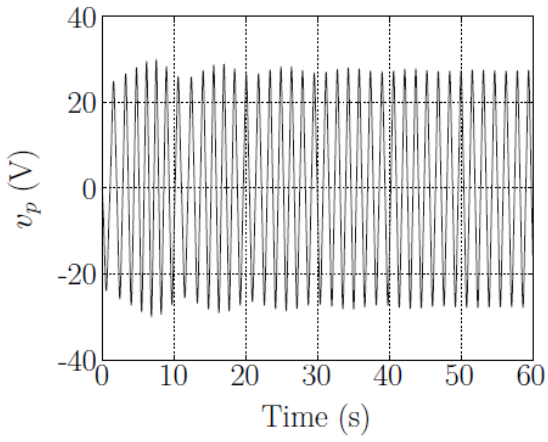

(a) Time voltage in PZT.

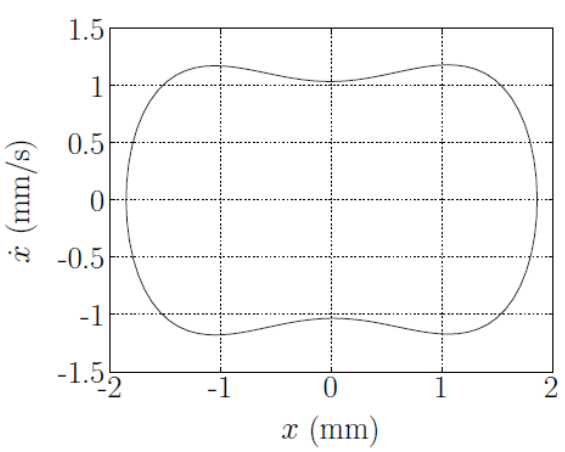

(b) Displacementxvelocity.

Figure 3: Theoretical analyses with $A=1.5 \mathrm{~g}$ and $\omega=50.27 \mathrm{rad} / \mathrm{s}$.

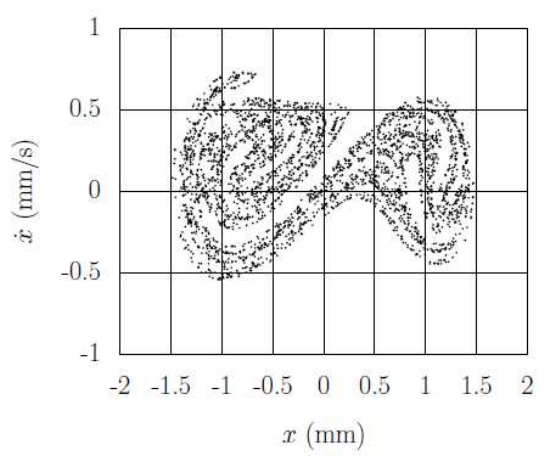

(a) $A=0.5 \mathrm{~g}$ and $\omega=50.27 \mathrm{rad} / \mathrm{s}$.

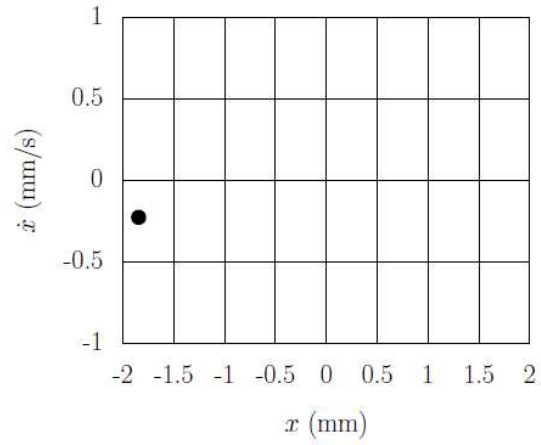

(b) $A=1.5 \mathrm{~g}$ and $\omega=50.27 \mathrm{rad} / \mathrm{s}$.

Figure 4: Poincaré mapping.

equations that describes the rectifier circuit (equations (8) and (10)).

Figure 6a presents the voltage tests redone considering the use of the rectifier circuit with the Poincaré map. If the initial conditions are changed the system changes its response, as seen in Figure 7.

Figure $8 \mathrm{a}$ shows the system is chaotic when $A=0.5 \mathrm{~g}$ and $\omega=50.27 \mathrm{rad} / \mathrm{s}$ and Figure $8 \mathrm{~b}$ presents a periodic motion, when $A=1.5 \mathrm{~g}$ and $\omega=50.27 \mathrm{rad} / \mathrm{s}$.

To analyze the behavior of the eletromechanical system, the Lyapunov ex- 


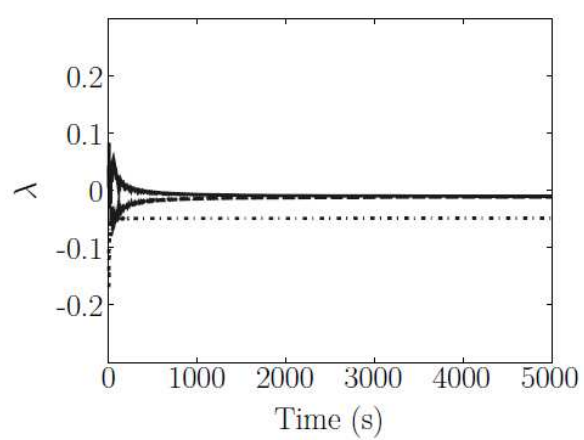

(a) $A=0.3 \mathrm{~g}$

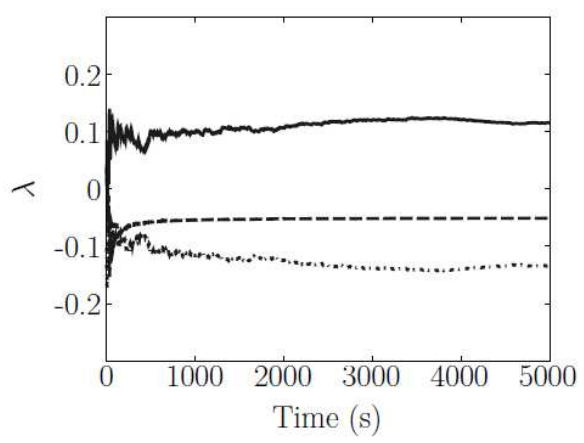

(c) $A=0.7 \mathrm{~g}$

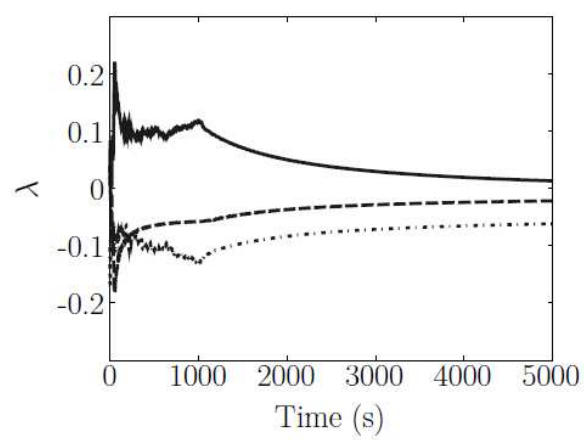

(b) $A=0.5 \mathrm{~g}$

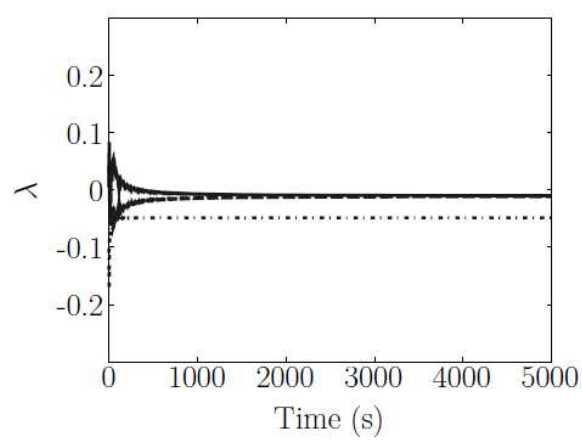

(d) $A=0.9 \mathrm{~g}$

Figure 5: Lyapunov exponents $\left.x, \dot{x}, v_{p}\right)$.

Table 2: Constitutive relations for the rectifier.

\begin{tabular}{cccc}
\hline Constitutive relation & Nomenclature & Value & Unit \\
\hline Resistance of the load & $R_{L}$ & 1000 & $\Omega$ \\
Capacitance of the capacitor & $C_{L}$ & 0.3 & $\mathrm{~F}$ \\
Resistance of diode & $R_{D}$ & 250 & $\Omega$ \\
\hline
\end{tabular}

ponents were again computed (Figure 9) and it can be seen that even coupled to rectifier circuit, the dynamical system also presents similar chaotic behavior for the same condition. 


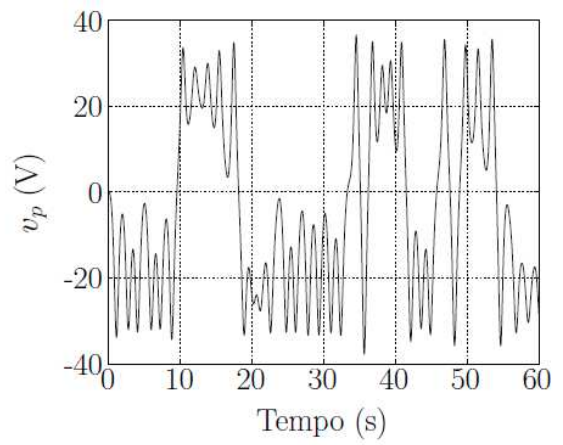

(a) Time voltage in PZT.

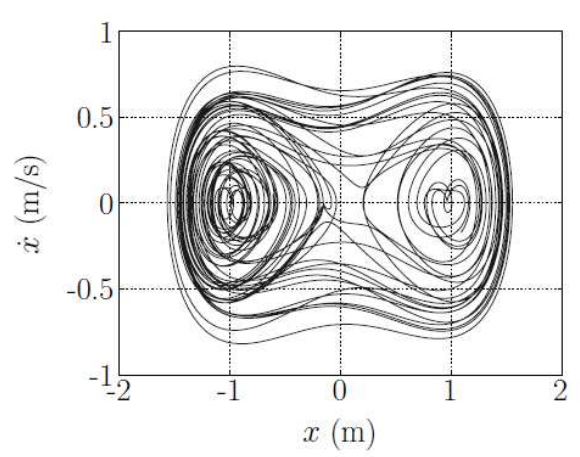

(b) Displacement×velocity.

Figure 6: Theoretical analyses with $A=0.5 \mathrm{~g}$ and $\omega=50.27 \mathrm{rad} / \mathrm{s}$.

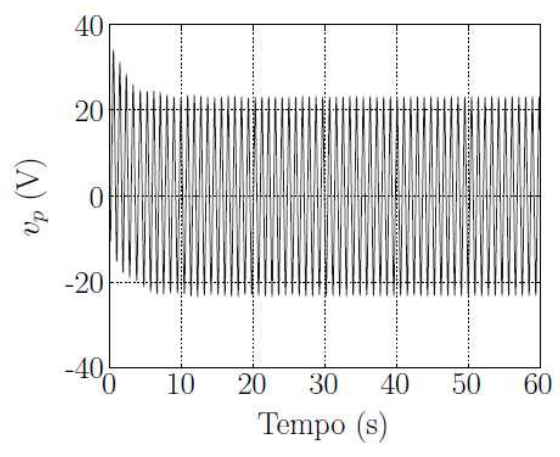

(a) Time voltage in PZT.

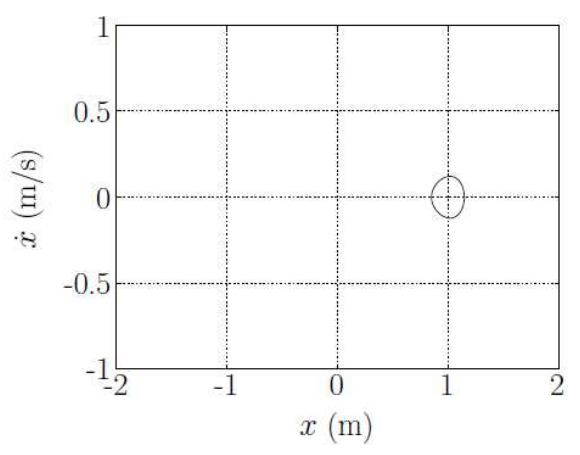

(b) Displacement×velocity.

Figure 7: Theoretical analyses with $A=1.5 \mathrm{~g}$ and $\omega=50.27 \mathrm{rad} / \mathrm{s}$.

Figure 10 presents the bifurcation plot varying amplitude and frequency of excitation for the mechanical device coupled with rectifier circuit. As can be seen, the device continues chaotic with and without the coupled rectifier. The points $A=0.3 \mathrm{~g}$ and $\omega=50.27 \mathrm{rad} / \mathrm{s}$ are associated with a bifurcation point, where the line splits in two thus replacing the stable fixed point. This sequence of bifurcations continues, featuring a cascade of doubling periods culminating in the emergence of chaos, characterized by a cloud of points in the diagram. 


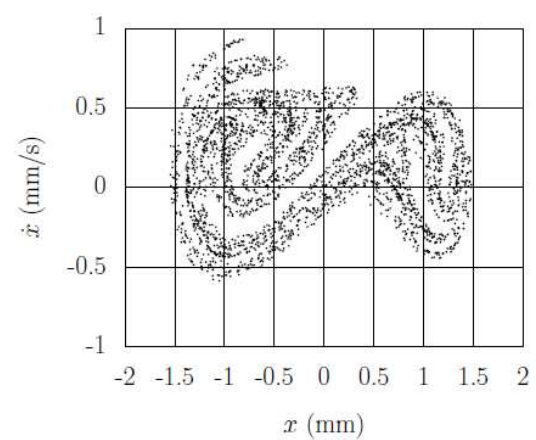

(a) $A=0.5 \mathrm{~g}$ and $\omega=50.27 \mathrm{rad} / \mathrm{s}$.

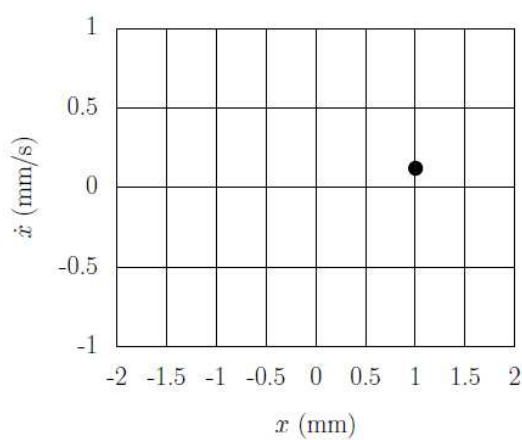

(b) $A=1.5 \mathrm{~g}$ and $\omega=50.27 \mathrm{rad} / \mathrm{s}$.

Figure 8: Poincaré mapping.

It can be seen that when coupled to the rectifier, the voltage generated by the PZT is higher for some values of frequency, $\omega$, and amplitude of excitation, $A$.

Finally, Figure 11 shows the rectified voltage to $\omega=50.26 \mathrm{rad} / \mathrm{s} R_{L}=10$ $\mathrm{k} \Omega$. Analyzing this figure, it can be seen that it is possible to rectify a chaotic voltage produced by the mechanical device. It is seen that a value outside the chaos region range, the system displays a lower rectified voltage value compared to when the system is in chaos.

\section{Final Remarks}

In this paper, a chaotic nonlinear energy harvesting coupled with a half-wave diode bridge rectifier is investigated through dynamic analysis. The Lyapunov exponents were calculated for time series and the results showed that the system is chaotic for certain values of amplitude and frequency of excitation. Even coupled with a rectification circuit, the device continues to present chaotic behavior under the same conditions. The presence of the bistability causes the system to rapidly switch between the stable states under proper mechanical excitations, thereby increasing the velocity and, in turn, the power converted by the harvester. However, the conditions for the device to undergo chaos are very specific, thereby making it very difficult for a practical application of this model. 


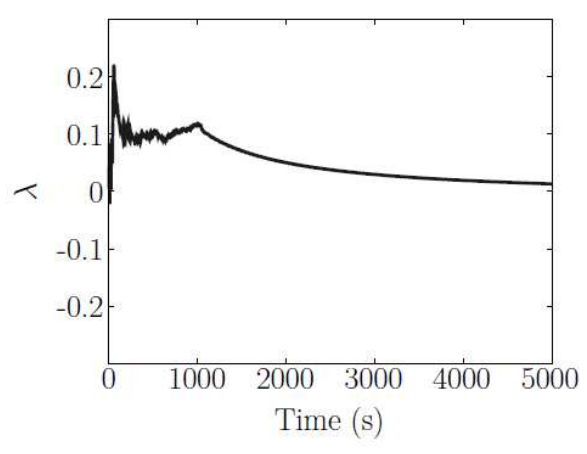

(a) $A=0.3 \mathrm{~g}$

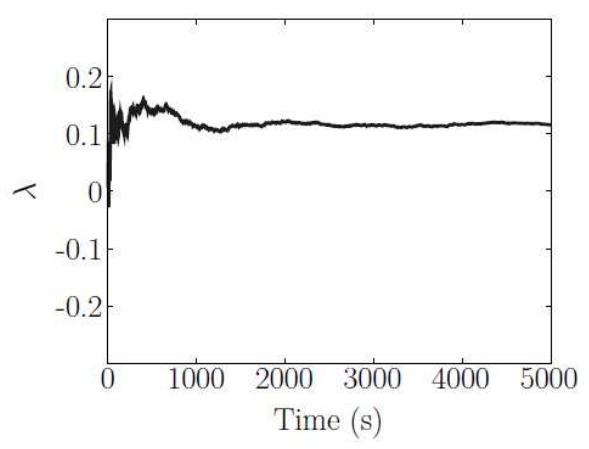

(c) $A=0.7 \mathrm{~g}$

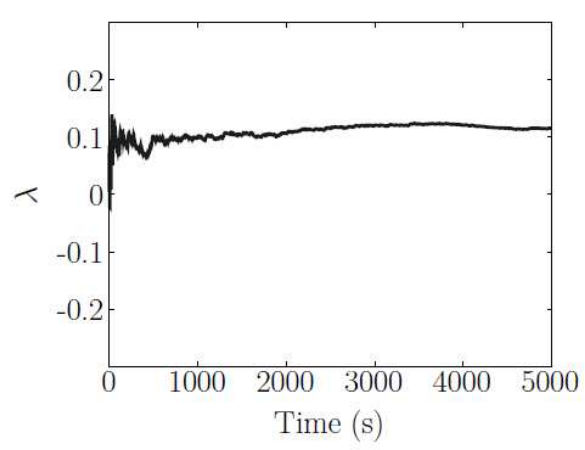

(b) $A=0.5 \mathrm{~g}$

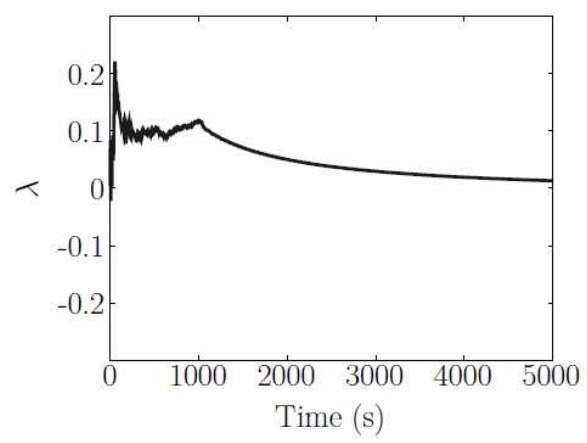

(d) $A=0.9 \mathrm{~g}$

Figure 9: Maximum Lyapunov exponents for time series.

\section{Acknowledgements}

The first author would like to thank the Coordenação de Aperfeiçoamento de Pessoal de Nível Superior (CAPES) for his scholarship. The authors are also grateful the financial support provided by the following to the Conselho Nacional de Desenvolvimento Científico e Tecnológico (CNPq) grant numbers 303403/2013-6 and 301769/2012-5 and also to the São Paulo Research Foundation (FAPESP) grant number 12/09135-3. 


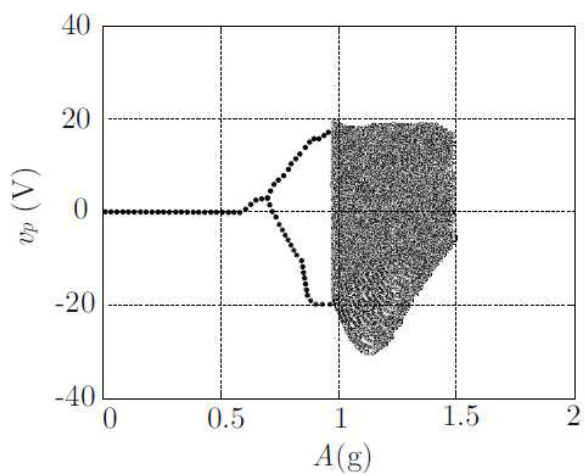

(a) Varying the amplitude

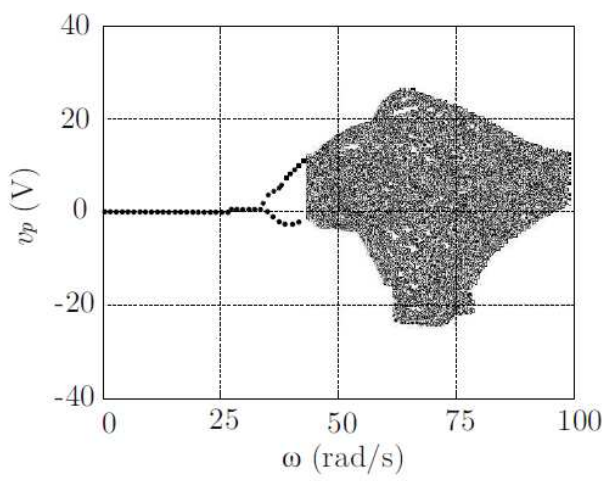

(b) Varying the frequency excitation

Figure 10: Bifurcation diagram for the nonlinear device.
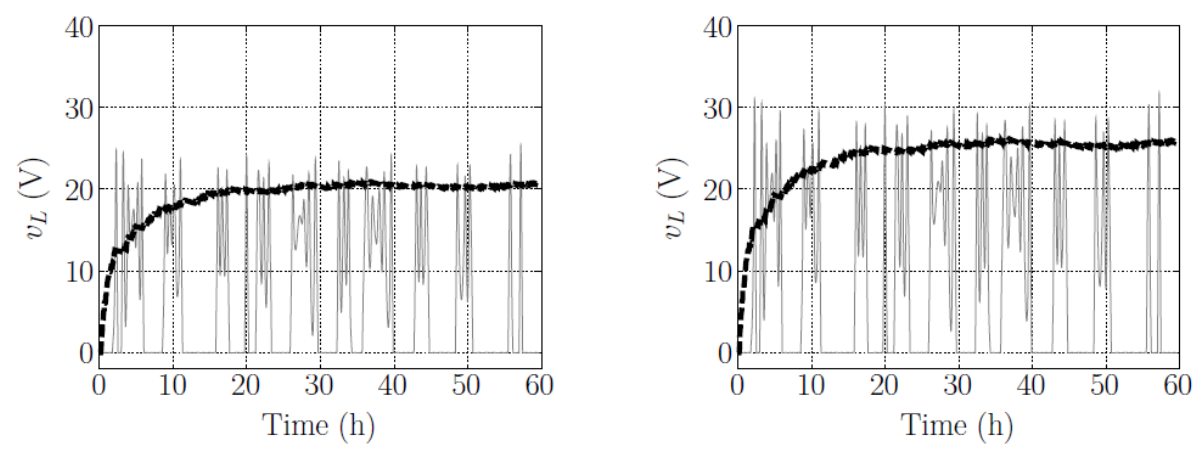

Figure 11: Rectified voltage to different values of amplitude.

\section{References}

[1] S. Roundy and P. K. Wright, A piezoelectric vibration based generator for wireless electronics, Smart Materials and structures, 13, No. 5 (2004), 1131. [DOI: http://dx.doi.org/10.1088/0964-1726/13/5/018]

[2] A. Abdelkefi and A. H. Nayfeh and M. R. Hajj, Design of piezoaeroelastic energy harvesters, Nonlinear Dynamics, 68, No. 4 (2012), 519-530. [DOI: http://dx.doi.org/10.1007/s11071-011-0233-x] 
[3] M. A. Karami and D. J. Inman, Equivalent damping and frequency change for linear and nonlinear hybrid vibrational energy harvesting systems, Journal of Sound and Vibration, 330, No. 23 (2011), 5583-5597. [DOI: http://dx.doi.org/10.1016/j.jsv.2011.06.021]

[4] M. El-Hami and P. Glynne-Jones and N. M. White M. and Hill and S. Beeby and E. James and A. D. Brown and J. N. Ross, Design and fabrication of a new vibration-based electromechanical power generator, Sensors and Actuators A: Physical, 92, No. 1 (2001), 335-342. [DOI: http://dx.doi.org/10.1016/S0924-4247(01)00569-6]

[5] F. Cottone and H. Vocca and L. Gammaitoni, Nonlinear energy harvesting, Physical Review Letters, 102, No. 8 (2009), 080601. [DOI: http://dx.doi.org/10.1103/PhysRevLett.102.080601]

[6] M. F. Daqaq and R. Masana and A. Erturk and D. D. Quinn, On the Role of Nonlinearities in Vibratory Energy Harvesting: A Critical Review and Discussion, Applied Mechanics Reviews, 66, No. 4 (2014), 040801. [DOI: http://dx.doi.org/10.1115/1.4026278]

[7] S. C. Stanton and C. C. McGehee and B. P. Mann, Nonlinear dynamics for broadband energy harvesting: Investigation of a bistable piezoelectric inertial generator, Physica D: Nonlinear Phenomena, 239, No. 10 (2010), 640-653. [DOI: http://dx.doi.org/10.1016/j.physd.2010.01.019]

[8] L. Tang and Y. Yang and C. K. Soh, Toward broadband vibration-based energy harvesting, Journal of Intelligent Material Systems and Structures, 21, No. 18 (2010), 1867-1897. [DOI: http://dx.doi.org/10.1177/1045389X10390249]

[9] A. Erturk and D. J. Inman, Issues in mathematical modeling of piezoelectric energy harvesters, Smart Materials and Structures, 17, No. 6 (2008), 065016. [DOI: http://dx.doi.org/10.1088/0964-1726/17/6/065016]

[10] A. Erturk and W. G. R. Vieira and C. de Marqui Jr and D. J. Inman, On the energy harvesting potential of piezoaeroelastic systems, Applied Physics Letters, 96, No. 18 (2010). [DOI: http://dx.doi.org/10.1063/1.3427405]

[11] X. D. Do and H. H. Nguyen and S. K. Han and S. G. Lee, A rectifier for piezoelectric energy harvesting system with series Synchronized Switch Harvesting Inductor, Solid-State Circuits Conference (A-SSCC), 2013 IEEE Asian. [DOI: http://dx.doi.org/10.1109/ASSCC.2013.6691034] 
[12] G. K. Ottman and H. F. Hofmann and A. C. Bhatt and G. A. Lesieutre, Adaptive piezoelectric energy harvesting circuit for wireless remote power supply, Power Electronics, IEEE Transactions on, 17, No. 5 (2002). [DOI: http://dx.doi.org/10.1109/TPEL.2002.802194]

[13] Y. K. Ramadass and A. P. Chandrakasan, An efficient piezoelectric energy harvesting interface circuit using a bias-flip rectifier and shared inductor, Solid-State Circuits, IEEE Journal of, 45, No. 1 (2010), 189-204. [DOI: http://dx.doi.org/10.1109/JSSC.2009.2034442]

[14] S. Naik and A. Phipps and I. Visarath and P. Cavaroc and A. MatusVargas and A. Palacios and H. G. Gonzalez-Hernandez, Energy harvesting with coupled magnetostrictive resonators, SPIE Smart Structures and Materials+ Nondestructive Evaluation and Health Monitoring, 2014.

[15] W. J. Su and J. Zu and Y. Zhu, Design and development of a broadband magnet-induced dual-cantilever piezoelectric energy harvester, Journal of Intelligent Material Systems and Structures, 1, No. 1 (2013). [DOI: http://dx.doi.org/10.1177/1045389X13498315]

[16] N. G. Elvin, Equivalent electrical circuits for advanced energy harvesting, Journal of Intelligent Material Systems and Structures, 1, No. 1 (2014). [DOI: http://dx.doi.org/10.1177/1045389X14521878]

[17] F. C. Moon, Chaotic vibrations: an introduction for applied scientists and engineers, Journal of Applied Mechanics, 128, No. 3 (1989), 535 - 536. [DOI: http://dx.doi.org/10.1115/1.3173762]

[18] A. Erturk and J. Hoffmann and D. J. Inman, A piezomagnetoelastic structure for broadband vibration energy harvesting, Applied Physics Letters, 94, No. 25 (2009). [DOI: http://dx.doi.org/10.1063/1.3159815]

[19] A. Erturk and D. J. Inman, Broadband piezoelectric power generation on high-energy orbits of the bistable Duffing oscillator with electromechanical coupling, Journal of Sound and Vibration, 330, No. 10 (2011). [DOI: http://dx.doi.org/10.1016/j.jsv.2010.11.018]

[20] H. Wang and Y. Tang and A. Khaligh, A bridgeless boost rectifier for low-voltage energy harvesting applications, IEEE transactions on power electronics, 28, No. 11 (2013). [DOI: http://dx.doi.org/10.1109/TPEL.2013.2242903] 
[21] Y. Y. Chen and D. Vasic and Y. P. Liu and F. Costa, Study of a piezoelectric switching circuit for energy harvesting with bistable broadband technique by work-cycle analysis, Journal of Intelligent Material Systems and Structures, 1, No. 1 (2012). [DOI: http://dx.doi.org/10.1177/1045389X12460339]

[22] W. Q. Liu and A. Badel and F. Formosa and Y. P. Wu and A. Agbossou, Novel piezoelectric bistable oscillator architecture for wideband vibration energy harvesting, Smart materials and structures, 22, No. 3 (2013). [DOI: http://dx.doi.org/10.1088/0964-1726/22/3/035013]

[23] M. A. Savi, Dinâmica não-linear e caos, Editora E-papers, Brasil (2006).

[24] A. Wolf and J. B. Swift and H. L. Swinney and J. A. Vastano, Determining Lyapunov exponents from a time series, Physica D: Nonlinear Phenomena, 16, No. 3 (1985). [DOI: http://dx.doi.org/10.1016/0167-2789(85)90011-9]

[25] M. A. Clementino and R. Reginatto and S. da Silva, Modeling of piezoelectric energy harvesting considering the dependence of the rectifier circuit, Journal of the Brazilian Society of Mechanical Sciences and Engineering, 36, No. 2 (2014). [DOI: http://dx.doi.org/10.1007/s40430-013-0070-6]. 
